equally important. Increasing violence and abuse toward HCWs should be taken seriously, and the offenders must be punished to prevent repeated offenses.

A well-planned and specific approach to address healthcare issues is needed, and implementation and follow-up are essential. The COVID-19 pandemic has reminded us all of the importance of HCWs and their role in providing a strong foundation for a country's health and well-being. As the Director-General of WHO, Dr. Tedros Adhanom Ghebreyesus, said, "No country, hospital or clinic can keep its patients safe unless it keeps its health workers safe."

Acknowledgments. None.

Financial support. No financial support was provided relevant to this article.

Conflicts of interest. All authors report no conflicts of interest relevant to this article.

\section{References}

1. India's struggle to track new COVID variants could worsen crisis. Times of India website. https://timesofindia.indiatimes.com/india/indias-struggle- to-track-new-covid-variants-could-worsen-crisis/articleshow/82426964.cms. Accessed May 17, 2021.

2. COVID-19 in India: why the second coronavirus wave is devastating. BBC News website. https://www.bbc.com/news/world-asia-india-56811315.amp. Accessed May 17, 2021.

3. Second wave of COVID-19 to take a toll on Indian healthcare system: Fitch Business-Standard News website. https://www.business-standard.com/article/ current-affairs/second-wave-of-covid-19-to-take-a-toll-on-indian-healthcaresystem-fitch-121041600297_1.html. Accessed May 17, 2021.

4. It's time we took hospital-acquired infections more seriously. The Wire website. https://science.thewire.in/health/its-time-we-took-hospitalacquired-infections-more-seriously/. Accessed May 17, 2021.

5. Khasne RW, Dhakulkar BS, Mahajan HC. Burnout among healthcare workers during the COVID-19 pandemic in India: results of a questionnaire-based survey. Indian J Crit Care Med 2020;24:664-671.

6. Keep health workers safe to keep patients safe. World Health Organization website. https://www.who.int/news/item/17-09-2020-keep-health-workerssafe-to-keep-patients-safe-who. Accessed May 17, 2021.

7. Parikh P, Mehta P, Bansal S, et al. Protecting healthcare professionals and workers (other than COVID-19 management facilities) from contamination during COVID-19 pandemic, March 26, 2020, in India. Indian J Med Sci 2020;72:3-4.

\title{
Influenza vaccination among healthcare personnel during the coronavirus disease 2019 (COVID-19) pandemic
}

\author{
Minji Kang MD ${ }^{1,3}$, Sherry Clark RN² , Sandra Mendoza ${ }^{2}$, Doramarie Arocha $\mathrm{PhD}^{3}$, James B. Cutrell MD ${ }^{1}$, \\ Trish M. Perl MD, MSc ${ }^{1}$ and Julie Trivedi MD ${ }^{1,3}$ \\ ${ }^{1}$ Division of Infectious Diseases and Geographic Medicine, Department of Medicine, University of Texas Southwestern Medical Center, Dallas, Texas, ${ }^{2}$ Department \\ of Occupational Health, University of Texas Southwestern Medical Center, Dallas, Texas and ${ }^{3}$ Infection Prevention, University of Texas Southwestern Medical \\ Center, Dallas, Texas
}

To the Editor-Influenza in healthcare personnel (HCP) may lead to absenteeism and nosocomial outbreaks. ${ }^{1,2}$ The Centers for Disease Control and Prevention, the Advisory Committee on Immunization Practices, and the Healthcare Infection Control Practices Advisory Committee recommend annual immunization of HCP. ${ }^{3}$ During the coronavirus disease 2019 (COVID-19) pandemic, influenza immunization was emphasized vigorously in hope of reducing the burden of respiratory illnesses, medical visits, and hospitalizations that would further strain the healthcare system. We compared influenza vaccine uptake and patterns of declination waivers among HCP before and during the COVID-19 pandemic, and we identified demographics or occupational roles that may be associated with influenza vaccine declination.

At University of Texas Southwestern Medical Center, all HCP are required to complete the annual Seasonal Flu Survey in ReadySet (Axion Health, Broomfield, CO), our employee health software database. On the annual seasonal flu survey, HCP can agree to receive the vaccination at mobile vaccination kiosks or occupational health clinics, upload documentation if vaccinated elsewhere, or decline vaccination by selecting yes or no to 1 or more commonly cited reason for declination. During the 2020-2021

\footnotetext{
Author for correspondence: Minji Kang, E-mail: Minji.kang@utsouthwestern.edu

Cite this article: Kang M, et al. (2022). Influenza vaccination among healthcare personnel during the coronavirus disease 2019 (COVID-19) pandemic. Infection Control \& Hospital Epidemiology, 43: 1303-1305, https://doi.org/10.1017/ice.2021.263
}

influenza season, additional resources such as video series, campus briefings, and town halls were utilized to highlight the importance of influenza vaccination to all HCP. In September, biweekly video series hosted by executive leadership were dedicated to the dual threats of COVID-19 and influenza. The president's biweekly campus briefings highlighted the importance of influenza vaccine, and the emergency operations center sent weekly e-mails to remind HCP of the ongoing influenza campaign.

HCP demographics, occupational role, influenza vaccine uptake, and reasons for declination were obtained for influenza seasons between 2019 and 2021. Data were analyzed using SPSS Statistics version 25 software (SPSS, Armonk, NY). Differences between means were compared using the Student $t$ test, and proportions were compared using $\chi^{2}$ tests. Variables that were statistically significant were analyzed by multivariate logistic regression. All tests were 2 -sided, and $P<.05$ was considered statistically significant. This study was exempt from institutional review board review under category 4 .

Vaccination uptake rate declined significantly during the COVID-19 pandemic, with 13,853 (55.7\%) HCP vaccinated, 1,027 (4.1\%) declined, and 10,010 (40.2\%) with incomplete surveys during the 2020-2021 influenza season compared with 15,757 (63.5\%) vaccinated, 664 (2.7\%) declined, and 8,393 (33.8\%) with incomplete surveys during the 2019-2020 influenza season $(P<.01)$. Safety and/or side effects were the most common reason 
Table 1. Seasonal Influenza Vaccination Compliance Rates, 2019-2021

\begin{tabular}{|c|c|c|c|c|c|c|c|c|}
\hline \multirow[b]{2}{*}{ Variable } & \multicolumn{4}{|c|}{ 2019-2020 } & \multicolumn{4}{|c|}{ 2020-2021 } \\
\hline & $\begin{array}{c}\text { Vaccinated } \\
\text { No. }(\%)\end{array}$ & $\begin{array}{l}\text { Declined } \\
\text { No. }(\%)\end{array}$ & $\begin{array}{l}\text { Odds Ratio } \\
(95 \% \mathrm{CI})\end{array}$ & $P$ Value & $\begin{array}{c}\text { Vaccinated } \\
\text { No. }(\%)\end{array}$ & $\begin{array}{l}\text { Declined } \\
\text { No. }(\%)\end{array}$ & $\begin{array}{l}\text { Odds Ratio } \\
(95 \% \mathrm{CI})\end{array}$ & $P$ Value \\
\hline Total & 15,757 & 664 & & & 13,853 & 1,027 & & \\
\hline Sex, female & $10,872(69.0)$ & $498(75)$ & $1.20(1.00-1.44)$ & .06 & 9,637 & 820 & $1.36(1.15-1.60)$ & $<.01$ \\
\hline Age, mean y $\pm S D$ & $41.2 \pm 12.4$ & $41.4 \pm 11.8$ & $1.00(1.00-1.01)$ & .43 & $41.8 \pm 12.4$ & $40.0 \pm 11.4$ & $0.99(0.98-0.99)$ & $<.01$ \\
\hline Occupation & & & & $<.01$ & & & & $<.01$ \\
\hline Nurses & $2,724(17.3)$ & $92(13.9)$ & Reference & & $2,468(17.8)$ & $192(18.7)$ & Reference & \\
\hline Physician, APP & $2,829(18.0)$ & $16(2.4)$ & $0.18(0.10-0.30)$ & & 2,645 (19.1) & $36(3.5)$ & $0.20(0.14-0.29)$ & \\
\hline Other clinical personnel & $3,280(20.8)$ & $142(21.4)$ & $1.31(1.00-1.71)$ & & $2,499(18.0)$ & $238(23.2)$ & $1.28(1.05-1.56)$ & \\
\hline Nutritional services & $212(1.3)$ & $25(3.8)$ & $3.58(2.25-5.70)$ & & $186(1.3)$ & $55(5.4)$ & $3.95(2.82-5.53)$ & \\
\hline Environmental services & $352(2.2)$ & $16(2.4)$ & $1.41(0.82-2.44)$ & & $360(2.6)$ & $17(1.7)$ & $0.71(0.42-1.18)$ & \\
\hline Administrative personnel & $3,147(20.0)$ & $216(32.5)$ & $2.04(1.59-2.62)$ & & $3,020(21.8)$ & $352(34.3)$ & 1.55 (1.29-1.86) & \\
\hline Other nonclinical personnel & $1,798(11.4)$ & $124(18.7)$ & $2.15(1.62-2.85)$ & & $1,399(10.1)$ & $118(11.5)$ & $1.17(0.92-1.49)$ & \\
\hline Research & $1,415(9.0)$ & $33(5.0)$ & $0.73(0.48-1.09)$ & & $1,276(9.2)$ & $19(1.9)$ & $0.20(0.12-0.32)$ & \\
\hline \multicolumn{9}{|l|}{ Declination reason } \\
\hline Medical reasons & \multicolumn{4}{|c|}{$68(10.2)$} & \multicolumn{4}{|c|}{$103(10.0)$} \\
\hline Safety/side effects & \multicolumn{4}{|c|}{$498(75.0)$} & \multicolumn{4}{|c|}{$702(68.4)$} \\
\hline Vaccine gives flu & \multicolumn{4}{|c|}{$256(38.6)$} & \multicolumn{4}{|c|}{$330(32.1)$} \\
\hline Vaccine doesn't prevent flu & \multicolumn{4}{|c|}{$361(54.4)$} & \multicolumn{4}{|c|}{$437(42.6)$} \\
\hline Religious reasons & \multicolumn{4}{|c|}{$172(25.9)$} & \multicolumn{4}{|c|}{$267(26.0)$} \\
\hline Fear of injections & \multicolumn{4}{|c|}{$121(18.2)$} & \multicolumn{4}{|c|}{$165(16.1)$} \\
\hline Not convenient & \multicolumn{4}{|c|}{$97(14.6)$} & \multicolumn{4}{|c|}{$134(13.0)$} \\
\hline Not important & \multicolumn{4}{|c|}{$139(20.9)$} & \multicolumn{4}{|c|}{$164(16.0)$} \\
\hline Other reasons & \multicolumn{4}{|c|}{$208(31.3)$} & \multicolumn{4}{|c|}{$276(26.9)$} \\
\hline
\end{tabular}

Note. SD, standard deviation; APP, advanced practice practitioners.

for declining, followed by "vaccine does not prevent flu," and "vaccine gives flu" (Table 1). In our multivariable analysis, occupational role was associated with influenza declination for both the 2019$2020(P<.01)$ and the $2020-2021$ influenza season $(P<.01)$. Physicians and advanced practice providers had low influenza declination rates (OR, 0.20; 95\% CI, 0.14-0.29), whereas personnel in nutrition services (OR, 3.95; 95\% CI, 2.82-5.53) and administrative personnel (OR, 1.55; 95\% CI, 1.29-1.86) had high declination rates (Table 1).

Despite efforts to raise awareness on the importance of influenza vaccination during the COVID-19 pandemic, our influenza vaccination rate declined significantly during the COVID-19 pandemic. The reason for this decline is unclear, but it is plausible that universal masking as well as increased number of HCP working from home may have contributed. Prior studies have reported various approaches to increasing influenza vaccination rates among $\mathrm{HCP}$ with varying success. ${ }^{4-6}$ Hospital policies requiring influenza vaccination among all HCP have been shown to dramatically improve vaccination rates. ${ }^{4}$ Nonmandatory strategies that involve education, peer-to-peer vaccination, mobile carts, use of declination forms, and prospective audit and feedback have been shown to improve vaccination uptake as well. ${ }^{5,6}$ Although reasons for declination included religious objections or medical reasons, the second and third most cited reasons for declination were related to the misinformation that vaccine causes influenza or that vaccine does not prevent influenza, confirming that even medically informed individuals are influenced by misinformation regarding vaccine efficacy and safety. ${ }^{7,8}$ Improving influenza vaccination uptake will require targeting common reasons for declining vaccination and tackling misinformation about vaccine efficacy and safety through educational interventions, peer advocacy, and prospective audit and feedback.

Although influenza vaccination uptake may not be directly reflective of the COVID-19 vaccine uptake, prior surveys have demonstrated that the most significant predictor for the acceptance of COVID-19 vaccine was acceptance of influenza vaccine. ${ }^{7,8}$ Although vaccine acceptance among $\mathrm{HCP}$ is higher than among non-HCP, vaccine skepticism and hesitancy are common among medical staff. ${ }^{7,8}$ In our study, HCP who are female, and work in administrative roles or in nutrition services had higher 
influenza vaccine declination rate for the 2020-2021 influenza season. This information may provide guidance on target groups that may benefit from vaccine education to alleviate apprehension regarding vaccine efficacy and safety.

This study has several limitations. A high proportion of HCP did not complete the annual seasonal flu survey. HCP who were preemployment, part-time workers, and/or contractors were included to complete the annual seasonal flu survey, and it is possible that they may have received their influenza vaccine elsewhere. Although race and ethnicity have been shown to be contributing factors to vaccine hesitancy, we were unable to obtain these data due to the limitations of our employee health system. Finally, the annual seasonal flu survey did not capture data for determining whether factors related to the COVID-19 pandemic may have contributed to the decline in influenza vaccination uptake and increase in incomplete surveys. It remains unclear whether the decline in influenza vaccination uptake will remain sustained in years to come, and additional studies will be necessary to determine the specific impact of COVID-19 on increased declination of influenza vaccination.

\section{Acknowledgments.}

Financial support. No financial support was provided relevant to this article.

\section{References}

1. Wilde JA, McMillan JA, Serwint J, et al. Effectiveness of influenza vaccine in healthcare professionals: a randomized trial. JAMA 1999;281:908-913.

2. Ahmed F, Lindley MC, Allred N, et al. Effect of influenza vaccination of healthcare personnel on morbidity and mortality among patients: systematic review and grading of evidence. Clin Infect Dis 2014;58:50-57.

3. Influenza vaccination information for health care workers. Centers for Disease Control and Prevention website. https://www.cdc.gov/flu/professionals/health careworkers.htm. Accessed January 5, 2021.

4. Quan K, Tehrani DM, Dickey L, et al. Voluntary to mandatory: evolution of strategies and attitudes toward influenza vaccination of healthcare personnel. Infect Control Hosp Epidemiol 2012;33:63-70.

5. Centers for Disease Control and Prevention (CDC). Interventions to increase influenza vaccination of health-care workers-California and Minnesota. Morb Mortal Wkly Rep 2005;54:196-199.

6. Honda H, Sato Y, Yamazaki A, et al. A successful strategy for increasing the influenza vaccination rate of healthcare workers without a mandatory policy outside of the United States: a multifaceted intervention in a Japanese tertiary care center. Infect Control Hosp Epidemiol 2013;34:1194-200.

7. Dror AA, Eisenbach N, Taiber S, et al. Vaccine hesitancy: the next challenge in the fight against COVID-19. Eur J Epidemiol 2020;35:775-779.

8. Gagneux-Brunon A, Detoc M, Bruel S, et al. Intention to get vaccinations against COVID-19 in French healthcare workers during the first pandemic wave: a cross-sectional survey. J Hosp Infect 2021;108:168-173. article.

\title{
Coronavirus disease 2019 (COVID-19) vaccinations and preservation of the healthcare workforce
}

\author{
Gabriela M. Andujar Vazquez ${ }^{1}$ (1), Jonathan Morely MPH${ }^{2}$, Helen W. Boucher MD ${ }^{1}$ and Shira I. Doron MD ${ }^{1}$ \\ ${ }^{1}$ Division of Geographic Medicine and Infectious Diseases, Department of Medicine, Tufts Medical Center, Boston, Massachusetts and ${ }^{2}$ Department of Emergency \\ Management, Tufts Medical Center, Boston, Massachusetts
}

To the Editor-Healthcare facilities have been stressed to their limits throughout the coronavirus disease 2019 (COVID-19) pandemic due to the combination of surges of patients and staffing shortages. US hospitals began vaccinating staff against COVID-19 exactly 4 months ago in mid-December 2020.

Tufts Medical Center, an urban 350-bed academic medical center with $\sim 7,400$ employees, began vaccinating staff with BNT162b2 vaccine (Pfizer) on December 16, 2020. On December 28,2020 , we incorporated the mRNA-1273 (Moderna) vaccine into our employee vaccination clinic. Gradually increasing eligibility to more groups, on January 26, 2021, eligibility was opened to all employees, including those working remotely. To date, 6,044 employees have been vaccinated with 2 doses of mRNA vaccine.

In total, 641 employees (including those working fully remotely), contractors, volunteers, interns, and students were

Author for correspondence: Gabriela M. Andujar Vazquez, E-mail: gandujarvazquez@ tuftsmedicalcenter.org

Cite this article: Andujar Vazquez GM, et al. (2022). Coronavirus disease 2019 (COVID-19) vaccinations and preservation of the healthcare workforce. Infection Control \& Hospital Epidemiology, 43: 1305-1306, https://doi.org/10.1017/ice.2021.238 infected with COVID-19 between March 16, 2020, and May 3,2021 . A precipitous drop in infections was observed after these healthcare workers began to receive their second doses of mRNA vaccine (Fig. 1). ${ }^{1}$ At its highest point, 90 employees were simultaneously out of work due to COVID-19-related illness. At the time of this writing, only 19 employees are out due to COVID-19, even though the incidence of COVID-19 remains high in the surrounding community.

We established a telephone hotline for employees, who were also encouraged to visit the employee health department as needed to report and discuss vaccine side effects. In total, 150 employees reported side effects, of which 12 met the criteria to be reported to the Vaccine Adverse Event Reporting System.

Our hospital continues to operate at or above capacity, not only due to COVID-19 admissions but also to the increasing acuity of illness in patients without COVID-19, possibly due to delays in care. Vaccination has enabled us to preserve our workforce and provide necessary care to patients. When first introduced, many healthcare workers, trusting the data that supported the emergency use authorization, signed up to be vaccinated despite what was for some a natural trepidation associated with the idea of a "new" 